\title{
A note on theta divisors of stable bundles
}

\author{
Sonia Brivio
}

\begin{abstract}
Let $C$ be a smooth complex irreducible projective curve of genus $g \geq 3$. We show that if $C$ is a Petri curve with $g \geq 4$, a general stable vector bundle $E$ on $C$, with integer slope, admits an irreducible and reduced theta divisor $\Theta_{E}$, whose singular locus has dimension $g-4$. If $C$ is non-hyperelliptic of genus 3, then actually $\Theta_{E}$ is smooth and irreducible for a general stable vector bundle $E$ with integer slope on $C$.
\end{abstract}

\section{Introduction}

Let $C$ be a smooth, irreducible, complex projective curve of genus $g \geq 3$, and let $\omega_{C}$ be the canonical line bundle on $C$. We recall that $C$ is said a Petri curve if for any line bundle $L$ on the curve, the Petri map, given by multiplication of sections,

$$
\mu_{L}: H^{0}(L) \otimes H^{0}\left(\omega_{C} \otimes L^{-1}\right) \rightarrow H^{0}\left(\omega_{C}\right)
$$

is injective, see [1].

Let $\mathcal{U}(r, d)$ denote the moduli space of $S$-equivalence classes of semistable vector bundles of rank $r \geq 2$ and degree $d$ on $C$. It is a normal irreducible projective variety of dimension $r^{2}(g-1)+1$. Except when $r=g=2$ or $r$ and $d$ are coprime, $\mathcal{U}(r, d)$ is singular and the open subset $\mathcal{U}(r, d)^{s} \subset \mathcal{U}(r, d)$ of smooth points corresponds to isomorphism classes of stable bundles. Moreover, $\mathcal{U}(r, d) \simeq \mathcal{U}\left(r, d^{\prime}\right)$ whenever $d^{\prime}-d=k r, \quad k \in \mathbb{Z}$. In particular, if $d=r(g-1)$ a natural Brill-Noether locus is defined as follows:

$$
\Theta_{r}=\left\{[E] \in \mathcal{U}(r, r(g-1)) \mid h^{0}(\operatorname{gr}(E)) \geq 1\right\},
$$

where $[E]$ denotes the $S$-equivalence class of $E$ and $\operatorname{gr}(E)$ is the polystable bundle defined by a Jordan-Hölder filtration of $E$, see [13]. Actually, $\Theta_{r}$ is an integral Cartier divisor, see [6], which is called theta divisor of $\mathcal{U}(r, r(g-1))$.

Mathematics Subject Classification (2010): Primary 14H60; Secondary 14H10.

Keywords: Vector bundles. 
For semistable vector bundles with integer slope we can introduce the notion of theta divisors, see [2]. Let $E$ be a semistable vector bundle on $C$ with integer slope $m=d / r \in \mathbb{Z}$. We set $h=g-1-m$. The tensor product defines a map:

$$
\tau: \mathcal{U}(r, r m) \times \operatorname{Pic}^{h}(C) \longrightarrow \mathcal{U}(r, r(g-1)),
$$

sending $([E], N) \rightarrow[E \otimes N]$. We can consider the pull-back $\tau^{*} \Theta_{r}$ of $\Theta_{r}$. When the intersection $\tau^{*} \Theta_{r} \cdot[E] \times \operatorname{Pic}^{h}(C)$ is proper, it defines an effective divisor $\Theta_{E}$ on $\operatorname{Pic}^{h}(C)$ which is called the theta divisor of $E$, see [12], [11], and [3], which is set theoretically:

$$
\Theta_{E}=\left\{N \in \operatorname{Pic}^{h}(C) \mid h^{0}(\operatorname{gr}(E) \otimes N) \geq 1\right\} .
$$

If $\operatorname{det} E \simeq M^{\otimes r}$, with $M \in \operatorname{Pic}^{m}(C)$, then it is well known that

$$
\Theta_{E} \in\left|r \Theta_{M}\right|,
$$

where $\Theta_{M}=\left\{N \in \operatorname{Pic}^{h}(C) \mid h^{0}(M \otimes N) \geq 1\right\}$, is a translate of the canonical theta divisor $\Theta \subset \mathrm{Pic}^{g-1}(C)$. Our result is the following:

Theorem 1.1. Let $r \geq 2$ and $m \in \mathcal{Z}$.

1. Let $C$ be a Petri curve of genus $g \geq 4$. For a general stable vector bundle $[E] \in \mathcal{U}(r, r m), \Theta_{E}$ is an irreducible and reduced divisor, whose singular locus

$$
\operatorname{Sing}\left(\Theta_{E}\right)=\left\{N \in \operatorname{Pic}^{h}(C) \mid h^{0}(E \otimes N) \geq 2\right\}, \quad h=g-1-m,
$$

has dimension $g-4$.

2. Let $C$ be a non-hyperelliptic curve of genus 3. A general stable vector bundle $[E] \in \mathcal{U}(r, r m)$ admits a smooth irreducible and reduced theta divisor $\Theta_{E}$.

The above description of $\operatorname{Sing}\left(\Theta_{E}\right)$ actually holds for a general stable bundle $E$ on any smooth curve, this is also proved with different arguments in [14], see also [5] for a generalization. Petri condition is required to prove the dimensional formula.

\section{Preliminary results}

Before proving our result we will recall some facts on the theta divisor $\Theta_{r}$ of the moduli space $\mathcal{U}(r, r(g-1))$. For any $k \geq 1$, we can define the following BrillNoether loci:

$$
\begin{aligned}
& B(r, r(g-1), k)=\left\{[F] \in \mathcal{U}(r, r(g-1))^{s} \mid h^{0}(F) \geq k\right\}, \\
& \tilde{B}(r, r(g-1), k)=\left\{[F] \in \mathcal{U}(r, r(g-1)) \mid h^{0}(g r(F)) \geq k\right\},
\end{aligned}
$$

which are closed subschemes of their moduli spaces. Note that

$$
\tilde{B}(r, r(g-1), 1)=\Theta_{r} .
$$

Moreover, we recall Laszlo's singularity theorem, see [8]:

Theorem 2.1. The multiplicity of $\Theta_{r}$ at a stable point $[F] \in \Theta_{r}$ is $h^{0}(F)$. 
This implies that

$$
B(r, r(g-1), 2)=\left\{[F] \in \mathcal{U}(r, r(g-1))^{s} \mid[F] \in \operatorname{Sing}\left(\Theta_{r}\right)\right\} .
$$

Brill-Noether loci have a determinantal description, which gives the following general results, see for instance [4] and [9].

Lemma 2.2. For $(r, r(g-1), k)$, the Brill-Noether number is the following:

$$
\beta(r, r(g-1), k)=r^{2}(g-1)+1-k^{2} .
$$

If $B(r, r(g-1), k)$ is not empty and $B(r, r(g-1), k) \neq \mathcal{U}(r, r(g-1))^{s}$, then we have the following properties:

(1) Every irreducible component of $B(r, r(g-1), k)$ has dimension $\geq \beta(r, r(g-$ $1), k)$;

(2) $B(r, r(g-1), k+1) \subset \operatorname{Sing}(r, r(g-1), k)$;

(3) The tangent space of $B(r, r(g-1), k)$ at a point $[F]$ with $h^{0}(F)=k$ can be identified with the dual of the cokernel of the Petri map, given by multiplication of sections:

$$
\mu_{F}: H^{0}(F) \otimes H^{0}\left(\omega_{C} \otimes F^{*}\right) \longrightarrow H^{0}\left(F \otimes F^{*} \otimes \omega_{C}\right)
$$

(4) $B(r, r(g-1), k)$ is smooth of dimension $\beta(r, r(g-1), k)$ at $[F]$ if and only if the Petri map $\mu_{F}$ is injective.

It is easy to produce semistable vector bundles $[F] \in \tilde{B}(r, r(g-1), k)$, however the non-emptiness of $B(r, r(g-1), k)$ is a more delicate question. For $k=2$ we have the following result:

Theorem 2.3. Let $C$ be a smooth curve of genus $g \geq 3$. Then for any $r \geq 2$, the Brill-Noether locus $B(r, r(g-1), 2)$ is non-empty.

This follows from a result of [10], which extends to arbitrary smooth curves the result of $[15]$.

As an application of the study of moduli spaces of coherent systems we have the following:

Theorem 2.4. Let $C$ be a Petri curve of genus $g \geq 3$ and $r \geq 2$. Then the Brill-Noether locus $B(r, r(g-1), 2)$ is irreducible of dimension

$$
\beta(r, r(g-1), 2)=r^{2}(g-1)-3=\operatorname{dim} \mathcal{U}(r, r(g-1))^{s}-4 .
$$

For the proof see [4], Theorem 11.11. 


\section{Proof of Theorem 1.1}

In this section we prove Theorem 1.1. Let $C$ be a smooth curve of genus $g \geq 3$. We will consider the map defined in (1.3):

$$
\tau: \mathcal{U}(r, r m) \times \operatorname{Pic}^{h}(C) \longrightarrow \mathcal{U}(r, r(g-1))
$$

sending $([E], N) \rightarrow[E \otimes N]$. Note that the restriction

$$
\tau_{N}=\tau_{\mid \mathcal{U}(r, r m) \times N}: \mathcal{U}(r, r m) \longrightarrow \mathcal{U}(r, r(g-1))
$$

is an isomorphism for any $N \in \mathrm{Pic}^{h}(C)$. We will consider the restriction of $\tau$ to stable bundles and we will denote it by $\tau_{s}$ :

$$
\tau_{s}: \mathcal{U}(r, r m)^{s} \times \operatorname{Pic}^{h}(C) \longrightarrow \mathcal{U}(r, r(g-1))^{s} .
$$

Note that $\tau_{s}$ is a smooth morphism whose fibers are biregular to $\operatorname{Pic}^{h}(C)$. Let $p_{i}, i=1,2$, denote the projections of $\mathcal{U}(r, r m)^{s} \times \operatorname{Pic}^{h}(C)$ onto factors. Let us consider the pull-back $\tau_{s}^{*} \Theta_{r}$ of $\Theta_{r}$ and the restriction of $p_{2}$ to $\tau_{s}^{*} \Theta_{r}$ :

$$
p_{2 \mid \tau_{s}^{*} \Theta_{r}}: \tau_{s}^{*} \Theta_{r} \longrightarrow \operatorname{Pic}^{h}(C) .
$$

It is a surjective map whose fibers are all isomorphic to $\Theta_{r \mid \mathcal{U}(r, r(g-1))^{s}}$, since the restriction $\tau_{s \mid \mathcal{U}(r, r m)^{s} \times N}$ is an isomorphism for any $N$. So all fibers are irreducible of the same dimension. This implies that $\tau_{s}^{*} \Theta_{r}$ is an irreducible subscheme of codimension 1 of $\mathcal{U}(r, r m)^{s} \times \operatorname{Pic}^{h}(C)$. Moreover, since $\tau_{s}$ is smooth, by (2.4) we have:

$$
\operatorname{Sing}\left(\tau_{s}^{*} \Theta_{r}\right)=\tau_{s}^{*}\left\{[F] \in \mathcal{U}(r, r(g-1))^{s} \mid[F] \in \operatorname{Sing}\left(\Theta_{r}\right)\right\}=\tau_{s}^{*} B(r, r(g-1), 2) .
$$

By Theorem 2.4, $B(r, r(g-1), 2)$ is an irreducible subscheme of $\mathcal{U}(r, r(g-1))^{s}$ of codimension 4. By the same argument used above, we can conclude that $\tau_{s}^{*} B(r, r(g-1), 2)$ is also an irreducible subscheme of $\mathcal{U}(r, r m)^{s} \times \operatorname{Pic}^{h}(C)$ of codimension 4 .

Let us consider the restriction of $p_{1}$ to $\tau_{s}^{*}\left(\Theta_{r}\right)$ :

$$
p_{1 \mid \tau_{s}^{*}\left(\Theta_{r}\right)}: \tau_{s}^{*}\left(\Theta_{r}\right) \longrightarrow \mathcal{U}(r, r m)^{s},
$$

for general $[E] \in \mathcal{U}(r, r m)^{s}$ the fiber at $[E]$ is actually the theta divisor $\Theta_{E}$. Let us consider the open subset of smooth points of $\tau_{s}^{*}\left(\Theta_{r}\right)$ :

$$
X=\tau_{s}^{*}\left(\Theta_{r}\right) \backslash \tau_{s}^{*} B(r, r(g-1), 2),
$$

and look at the restriction of $p_{1}$ to $X$ :

$$
p_{1 \mid X}: X \longrightarrow \mathcal{U}(r, r m)^{s} .
$$

It is a dominant map, moreover since $X$ and $\mathcal{U}(r, r m)^{s}$ are smooth and irreducible, by generic smoothness, see [7], there exists an open subset

$$
V \subset \mathcal{U}(r, r m)^{s}
$$


such that $p_{1 \mid X \cap p_{1}{ }^{-1}(V)}: X \cap p_{1}{ }^{-1}(V) \rightarrow V$ is a smooth morphism. This implies that for $[E] \in V$ the fiber $\Theta_{E} \cap X$ is smooth, that is,

$$
\operatorname{Sing}\left(\Theta_{E}\right)=\Theta_{E} \cdot \tau_{s}^{*} B(r, r(g-1), 2)=\left\{N \in \operatorname{Pic}^{h}(C) \mid h^{0}(E \otimes N) \geq 2\right\} .
$$

By the above considerations, we can conclude that for a general vector bundle $[E] \in \mathcal{U}(r, r m)^{s}$, the divisor $\Theta_{E}$ is irreducible and reduced, and its singular locus is described in (3.5). So Theorem 1.1 follows from the next two lemmas.

Lemma 3.1. Let $C$ be a non-hyperelliptic curve of genus 3. Then, for a general stable $[E] \in \mathcal{U}(r, r m)^{s}$, the intersection $\Theta_{E} \cdot \tau_{s}^{*} B(r, 2 r, 2)$ is empty.

Proof. If $C$ is non-hyperelliptic of genus 3 then

$$
\operatorname{dim} \tau_{s}^{*} B(r, 2 r, 2)=2 r^{2}<\operatorname{dim} \mathcal{U}(r, r m)^{s}=2 r^{2}+1 .
$$

Let us consider the restriction of $p_{1}$ to $\tau_{s}^{*} B(r, 2 r, 2)$ :

$$
p_{1 \mid \tau_{s}^{*} B(r, 2 r, 2)}: \tau_{s}^{*} B(r, 2 r, 2) \longrightarrow \mathcal{U}(r, r m)^{s} .
$$

The image of $p_{1 \mid \tau_{s}^{*} B(r, 2 r, 2)}$ is a closed subvariety of $\mathcal{U}(r, r m)^{s}$, so we can conclude for general $[E] \in \mathcal{U}(r, r m)^{s}$ the intersection $\Theta_{E} \cdot \tau_{s}^{*} B(r, 2 r, 2)$ is empty.

Lemma 3.2. Let $C$ be a Petri curve of genus $g \geq 4$. Then, for a general stable $[E] \in \mathcal{U}(r, r m)^{s}$, the intersection $\Theta_{E} \cdot \tau_{s}^{*} B(r, r(g-1), 2)$ has dimension $g-4$.

Proof. Let us consider the restriction of $p_{1}$ to $\tau_{s}^{*} B(r, r(g-1), 2)$ :

$$
p_{1 \mid \tau_{s}^{*} B(r, r(g-1), 2)}: \tau_{s}^{*} B(r, r(g-1), 2) \longrightarrow \mathcal{U}(r, r m)^{s} .
$$

We prove that the map (3.7) is dominant, hence a general fiber has dimension $g-4$.

For $([E], N) \in \mathcal{U}(r, r m)^{s} \times \operatorname{Pic}^{h}(C)$ let $d\left(p_{1}\right)_{[E], N}$ be the induced map on tangent spaces:

$$
d\left(p_{1}\right)_{[E], N}: T_{[E]}(\mathcal{U}(r, r m)) \times T_{N}\left(\operatorname{Pic}^{h}(C)\right) \longrightarrow T_{[E]}(\mathcal{U}(r, r m)),
$$

whose kernel is the tangent space $T_{[E], N}\left([E] \times \operatorname{Pic}^{h}(C)\right)$ of the fiber of $p_{1}$ at $[E]$. For a general $([E], N) \in \tau_{s}^{*} B(r, r(g-1), 2)$, let us consider the restriction

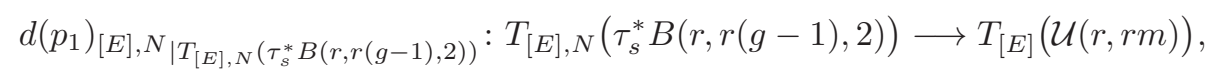

it is a surjective map if and only we have:

$$
\operatorname{dim} T_{[E], N}\left(\tau_{s}^{*} B(r, r(g-1), 2)\right) \cap T_{[E], N}\left([E] \times \operatorname{Pic}^{h}(C)\right)=g-4 .
$$

We recall that for any $([E], N) \in \mathcal{U}(r, r m)^{s} \times \operatorname{Pic}^{h}(C)$ we have:

$$
\begin{aligned}
T_{[E], N}\left([E] \times \operatorname{Pic}^{h}(C)\right) & \simeq H^{1}\left(O_{C}\right), \\
T_{[E], N}\left(\tau_{s}^{*} B(r, r(g-1), 2)\right) & =\tau_{s}^{*}\left(T_{[E \otimes N]}(B(r, r(g-1), 2))\right) .
\end{aligned}
$$


Finally if $h^{0}(E \otimes N)=2$, by Lemma 2.2, we have:

$$
T_{[E \otimes N]}(B(r, r(g-1), 2)) \simeq\left(\operatorname{coker} \mu_{E \otimes N}\right)^{*} \subset H^{1}\left(E \otimes E^{*}\right),
$$

where $\mu_{E \otimes N}$ is the Petri map of $E \otimes N$, and

$$
H^{1}\left(E \otimes E^{*}\right) \simeq T_{[E]}(\mathcal{U}(r, r m)) \simeq T_{[E \otimes N]}(\mathcal{U}(r, r(g-1))) .
$$

We also recall that $E \otimes E^{*}$ is the sheaf of endomorphisms of $E$ and the trace homomorphism $t r$ defines the subsheaf of tracenull endomorphisms:

$$
0 \rightarrow \operatorname{End}_{0}(E) \rightarrow E \otimes E^{*} \rightarrow O_{C} \rightarrow 0 .
$$

If $E$ is stable then $H^{0}\left(E \otimes E^{*}\right)=\left\{\lambda \cdot \operatorname{id}_{E} \mid \lambda \in \mathcal{C}\right\} \simeq H^{0}\left(O_{C}\right)$, so that we have

$$
H^{1}\left(E \otimes E^{*}\right)=H^{1}\left(\operatorname{End}_{0}(E)\right) \oplus H^{1}\left(O_{C}\right) ;
$$

moreover, $H^{1}\left(O_{C}\right)$ is the image of the tangent space of $[E] \times \operatorname{Pic}^{h}(C)$ :

$$
d\left(\tau_{E}\right)_{N}: H^{1}\left(O_{C}\right) \longrightarrow H^{1}\left(E \otimes E^{*}\right) .
$$

So to prove (3.8), it is enough to prove that for a general stable vector bundle $E \otimes N \in B(r, r(g-1), 2)$ we have that

$$
\operatorname{dim}\left(H^{1}\left(O_{C}\right) \cap\left(\operatorname{coker} \mu_{E \otimes N}\right)^{*}\right)=g-4 .
$$

By taking dual spaces of (3.10), let

$$
\pi_{E}: H^{0}\left(E \otimes E^{*} \otimes \omega_{C}\right) \longrightarrow H^{0}\left(\omega_{C}\right)
$$

be the natural projection map. Then condition (3.12) is satisfied if and only if we require that the composition of the Petri map $\mu_{E \otimes N}$ with $\pi_{E}$ has maximal rank:

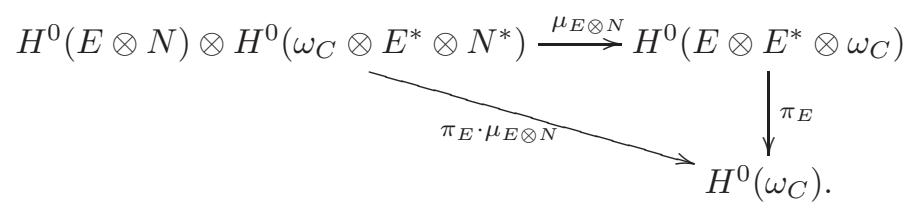

Note that actually $\pi_{E}$ can be identified with the map induced on global sections from the exact sequence (3.9) tensored with the canonical line bundle $\omega_{C}$.

Finally, we prove by induction on $r$ that the map $\pi_{g r F} \cdot \mu_{g r F}$ has maximal rank for a general $[F] \in \tilde{B}(r, r(g-1), 2)$.

Let $r=1$ : for a general line bundle $L \in B(1, g-1,2)=W_{g-1}^{1}$, the Petri map is injective

$$
\mu_{L}: H^{0}(L) \otimes H^{0}\left(\omega_{C} \otimes L^{*}\right) \longrightarrow H^{0}\left(\omega_{C}\right)
$$

since $C$ is Petri, and the map $\pi_{L}$ is the identity.

Let $G \in B(r-1,(r-1)(g-1), 2)$ be a general stable bundle satisfying the claim. Let us consider the following semistable vector bundle:

$$
F=G \oplus L, \quad L \in \operatorname{Pic}^{g-1}(C), \quad h^{0}(L)=0 .
$$


Then $H^{0}(F) \simeq H^{0}(G)$ and $H^{0}\left(\omega_{C} \otimes F^{*}\right) \simeq H^{0}\left(\omega_{C} \otimes G^{*}\right)$, so we can conclude that $[F] \in \tilde{B}(r, r(g-1), 2)$. Let $i: H^{0}\left(G \otimes G^{*} \otimes \omega_{C}\right) \hookrightarrow H^{0}\left(F \otimes F^{*} \otimes \omega_{C}\right)$ be the natural inclusion. We have the following commutative diagram:

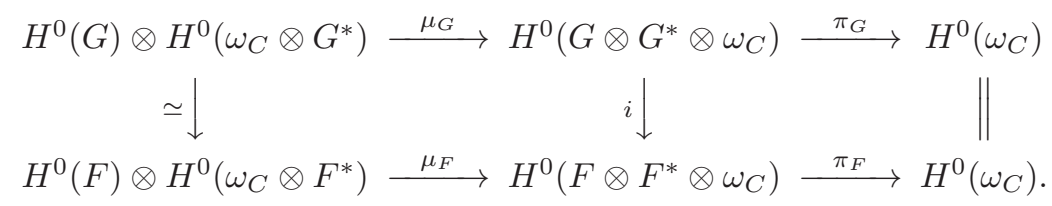

By induction hypothesis the composition map $\pi_{G} \cdot \mu_{G}$ has maximal rank, so $\pi_{F} \cdot \mu_{F}$ has maximal rank too. Let $U \subset \tilde{B}(r, r(g-1), 2)$ be the subset corresponding to classes $[F]$ such that the map $\pi_{g r F} \cdot \mu_{g r F}$ has maximal rank, $U$ is not empty. Since this condition is open on each family of vector bundles of $\tilde{B}(r, r(g-1), 2)$, it follows that $U$ is a non empty open subset of $\tilde{B}(r, r(g-1), 2)$. This implies that a general $[F] \in \tilde{B}(r, r(g-1), 2)$ satisfies the property too.

This concludes the proof of Theorem 1.1.

Acknowledgements. I am grateful to Alessandro Verra and Michele Bolognesi for many discussions on the matter.

\section{References}

[1] Arbarello, E., Cornalba, M. and Griffiths, P.A.: Geometry of algebraic curves, Vol. II. Grundlehren der Mathematischen Wissenschaften 268, Springer, Heidelberg, 2011.

[2] Beauville, A.: Some stable vector bundles with reducible theta divisors. Manuscripta Math. 110 (2003), no. 3, 343-349.

[3] Beauville, A., Narasimhan, M. S. and Ramanan, S.: Spectral curves and the generalized theta divisor. J. Reine Angew. Math 398 (1989), 169-179.

[4] Bradlow, S. B., García-Prada, O., Muñoz, V. and Newstead, P. E.: Coherent systems and Brill-Noether theory. Internat. J. Math. 14 (2003), no. 7, 683-733.

[5] Casalaina-Martin, S. And Teixidor i Bigas, M.: Singularities of Brill-Noether loci for vector bundles on a curve. Math. Nachr. 284 (2011), no. 14-15, 1846-1871.

[6] Drezet J. M. and Narasimhan, M. S.: Groupe de Picard des variétés de modules de fibrés semi-stables sur les courbes algébriques. Invent. Math. 97 (1989), no. 1, 53-94.

[7] Hartshorne, R.: Algebraic geometry. Graduate texts in Mathematics 52, SpringerVerlag, New York-Heidelberg, 1977.

[8] Laszlo, Y.: Un théorème de Riemann pour les diviseurs thêta sur les espaces de modules de fibrés stables sur une courbe. Duke Math. Journ. 64 (1991), no. 2, 333-347.

[9] Mercat, V.: Le probléme de Brill-Noether: présentation, 2001. Available at http://www.liv.ac.uk / newstead/presen/presenta.ps.

[10] Mercat, V.: Le probléme de Brill-Noether et le theoréme de Teixidor. Manuscripta Math. 98 (1999), no. 1, 75-85. 
[11] Narasimhan, M.S. and Ramanan, S.: Moduli of vector bundles on a compact Riemann surface. Ann. of Math. (2) 89 (1969), 14-51.

[12] Narasimhan, M.S. And Ramanan, S.: Vector bundles on curves. In Algebraic geometry (Internat. Colloq., Tata Inst. Fund. Res., Bombay, 1968), 335-346. Oxford Univ. Press, London, 1969.

[13] Seshadri, C. S.: Fibrés vectoriels sur les courbes algébriques. Asterisque 96, Société Mathématique de France, Paris, 1982.

[14] Teixidor i Bigas, M.: A Riemann singularity theorem for generalised Brill-Noether loci. Math. Nachr. 196 (1998), 251-257.

[15] Teixidor I Bigas, M.: Brill-Noether theory for stable vector bundles. Duke Math. J. 62 (1991), no. 2, 385-400.

Received May 14, 2013.

Sonia Brivio: Dipartimento di Matematica "F. Casorati", Universitá di Pavia, Via Ferrata 1, 27100 Pavia, Italy.

E-mail: sonia.brivio@unipv.it

Partially supported by PRIN 2009: "Moduli, strutture geometriche e loro applicazioni". 